\title{
COMPUTER-ASSISTED PROOFS OF SPECIAL FUNCTION IDENTITIES RELATED TO POISSON INTEGRALS
}

\author{
FLAVIA STAN \\ RESEARCH INSTITUTE FOR SYMBOLIC COMPUTATION (RISC) \\ JOHANNES KEPLER UNIVERSITY, 4040 LINZ, AUSTRIA
}

Using classical analytic methods, E. Symeonidis [5] derived explicit expressions for the Poisson kernels of geodesic balls in non-euclidean spaces. As a by-product of his work, Symeonidis obtained indirect proofs of two interesting special function identities involving Gegenbauer polynomials. In [3] the question of direct proofs was posed. We answer this question by presenting direct proofs obtained with the help of computer algebra algorithms based on WZ theory.

\section{INTRODUCTION}

The following two special function identities were obtained in [5] while computing expressions of the Poisson kernel for geodesic balls in the cases of the spheres and real hyperbolic spaces of arbitrary dimension. By showing that the two sides of each identity are expressing one and the same Poisson kernel, E. Symeonidis has proven the following two theorems.

Theorem 1. For real $x$ and $t$ such that $|x|<1,|t|<1$ and $n \in \mathbb{N}$ with $n \geq 3$,

$$
\sum_{k \geq 0} \frac{\left(\begin{array}{c}
k+n-2 \\
k
\end{array}\right)}{\left(\begin{array}{c}
k+\frac{n}{2}-2 \\
k
\end{array}\right)} t_{2}^{k} F_{1}\left(\begin{array}{c}
k, 1-\frac{n}{2} \\
k+\frac{n}{2}
\end{array} ; t^{2}\right) C_{k}^{\frac{n-2}{2}}(x)=\left(\frac{1-t^{2}}{1-2 t x+t^{2}}\right)^{n-1}
$$

Theorem 2. For real $x$ and $t$ such that $|x|<1,|t|<1$ and $n \in \mathbb{N}$ with $n \geq 3$,

$$
\begin{array}{r}
\sum_{k \geq 0} \frac{\Gamma\left(\frac{k+n}{2}\right) \Gamma\left(\frac{k+1}{2}\right)}{\Gamma\left(\frac{n}{2}+k-1\right)} t^{k}{ }_{2} F_{1}\left(\begin{array}{c}
k, k+n-1 \\
k+\frac{n}{2}
\end{array} ; \frac{1-\sqrt{1-t^{2}}}{2}\right) C_{k}^{\frac{n-2}{2}}(x)= \\
=\frac{(n-2) \Gamma\left(\frac{n+1}{2}\right)}{2 \Gamma\left(\frac{n}{2}+1\right)} \sqrt{1-t^{2}}{ }_{2} F_{1}\left(\begin{array}{c}
n, 1 \\
\frac{n}{2}+1
\end{array} ; \frac{x t+1}{2}\right) .
\end{array}
$$

In the above theorems, the following notation has been used to denote the ultraspherical or Gegenbauer polynomials ([1], 6.4.12),

$$
C_{k}^{\lambda}(x)=\frac{(\lambda)_{k}}{\Gamma(k+1)}(2 x)^{k}{ }_{2} F_{1}\left(\begin{array}{c}
-\frac{k}{2}, \frac{1-k}{2} \\
1-k-\lambda
\end{array} ; \frac{1}{x^{2}}\right) .
$$

Independent of Symeonidis' original derivation and of the background of the identities we present a direct approach based on computer algebra methods which is easy to follow and could be applied when proving other similar identities.

The basic idea for our proofs is to transform the problem into that of proving equality of sequences of coefficients defined by multiple sums. Then, such multisum identities are proven

Date: January 31, 2008.

Supported by SFB grant F1301 of the Austrian Science Foundation FWF. 
by finding a recurrence that is satisfied by both sides of the identity and by checking the equality of finitely many initial values. The structure of the identities in question will allow us the use of computer algebra algorithms to compute the necessary recurrences.

By Taylor expansion around the origin, any side of the identities (1) and (2) can be rewritten as

$$
\sum_{i, j \geq 0} c_{i, j}(\mu) t^{i} x^{j}
$$

where the coefficients $c_{i, j}(\mu)$ are multiple sums of the form

$$
c_{i, j}(\mu)=\sum_{\kappa_{1}} \cdots \sum_{\kappa_{r}} F_{i, j}\left(\mu, \kappa_{1}, \ldots, \kappa_{r}\right),
$$

and the summands $F_{i, j}\left(\mu, \kappa_{1}, \ldots, \kappa_{r}\right)$ are hypergeometric terms in all integer variables $\mu_{n}$ from $\mu=\left(\mu_{1}, \ldots, \mu_{p}\right)$ and in all summation variables $\kappa_{l}$ from $\kappa=\left(\kappa_{1}, \ldots, \kappa_{r}\right)$.

Remark: Recall that an expression $\mathcal{F}(\mu, \kappa)$ is called hypergeometric $[8,7]$ if there exists a rational function $R(\mu, \kappa)$ such that $\frac{\mathcal{F}(\mu, \kappa)}{\mathcal{F}(\mu-m, \kappa-k)}=R(\mu, \kappa)$ at the points $m \in \mathbb{Z}^{p}$ and $k \in \mathbb{Z}^{r}$ where this ratio is defined.

Under these conditions, for multiple sums of the form (5) or of the more general form (4), recurrences in even more than one variable $\mu_{n}$ can be found, for instance, using the Mathematica implementation of Wegschaider's algorithm [6] which is an extension of multivariate WZ summation [7].

$\ln [1]:=<<$ MultiSum.m

MultiSum Package by Kurt Wegschaider (enhanced by Axel Riese and

Burkhard Zimmermann) - (C) RISC Linz - V2.02 $\beta$ (02/21/05)

Given a term $\mathcal{F}(\mu, \kappa)$ which is hypergeometric in all parameters and a set of shifts $\mathbb{S} \subset \mathbb{Z}^{p+r}$, the algorithm [6] first computes a certificate recurrence of the form

$$
\sum_{(m, k) \in \mathbb{S}} a_{m, k}(\mu) \mathcal{F}(\mu-m, \kappa-k)=\sum_{l=1}^{r} \Delta_{\kappa_{l}}\left(R_{l}(\mu, \kappa) \mathcal{F}(\mu, \kappa)\right),
$$

where $a_{m, k}(\mu)$ are polynomials, not all zero, and $R_{l}(\mu, \kappa)$ are rational functions.

Note that the forward shift operators $\Delta_{\kappa_{l}}$ are defined as

$$
\Delta_{\kappa_{l}} \mathcal{F}(\mu, \kappa):=\mathcal{F}\left(\mu, \kappa_{1}, \ldots, \kappa_{l}+1, \ldots, \kappa_{r}\right)-\mathcal{F}(\mu, \kappa) .
$$

By summing over the certificate recurrence (6), we obtain a recurrence for sums of the form (5) because the coefficients $a_{m, k}(\mu)$ are free of the summation variables from $\kappa$ and the $\Delta$-parts on the right hand side telescope.

However, when we want to pass from the certificate recurrences (6) to recurrences for infinite sums over some parameters $\kappa_{l}$ from $\kappa$, we have to study the behaviour of expressions of the form $R_{l}(\mu, \kappa) \mathcal{F}(\mu, \kappa)$ when the parameter $\kappa_{l}$ tends to infinity. Only after these limit considerations we can decide if the recurrence for the sum $\sum_{\kappa} \mathcal{F}(\mu, \kappa)$ is homogeneous. Throughout the proofs of the above theorems, we will always check the homogeneity of the recurrences we have computed algorithmically.

Wegschaider's algorithm [6] determines recurrences, after making an Ansatz about their structure (i.e., fixing the set $\mathbb{S}$ of shifts that they contain), by solving a large system of equations over a field of rational functions. If the input of the algorithm is involved, computations will be time consuming; in addition, we might find only high order recurrences which means 
many initial values need to be checked. Consequently, directly applying this algorithm for large and intricately nested multiple sums of the more general type (4), for example, such as the ones appearing in the identities (1) and (2), is not advisable in practice.

Moreover, the initial values for such output recurrences might again be complicated sums. In this case, the algorithm can be applied again, provided that for these new identities an independent variable $\mu_{n}$ from $\mu$ is left. Iterating this procedure, we will end up with single sum representations of initial values that still need to be proven. If at this last step, recurrences in a single parameter $\mu_{n}$ are sufficient, one can use Zeilberger's algorithm [8] .

To avoid involved computations, before searching algorithmically for recurrences, we apply coefficient comparison with respect to the additional real variables $x$ and $t$ and we end up with identities whose sides are of type (5). This is a well-known strategy to eliminate summation quantifiers and to reduce the number of variables. Coefficient comparisons might lead to case distinctions, but in either case, the input for the algorithm [6] becomes significantly smaller. In this way, we reduce the identity (2) to a single summation problem and use the implementation [4] of Zeilberger's algorithm which is more efficient than the one described by Wegschaider in [6].

Remark: Another advantage of coefficient comparison is that it introduces "useful" discrete variables. For instance, in view of (4), if we compare coefficients with respect to $t^{i} x^{j}$, these arbitrarily chosen powers $i, j \in \mathbb{Z}$ will be further independent variables in addition to those of $\mu$. Furthermore, the recurrences in these new variables often are of low order, so the initial values are easier to check.

Remark: In order to keep proofs readable, we sometimes use the notation

$$
\left.\mathcal{F}(\mu, \kappa)\right|_{\kappa_{l}=q}:=\mathcal{F}\left(\mu, \kappa_{1}, \ldots, \kappa_{l-1}, q, \kappa_{l+1}, \ldots, \kappa_{r}\right)
$$

where $q \in \mathbb{Z}$ and, as above, $\kappa=\left(\kappa_{1}, \ldots, \kappa_{r}\right)$ and $\mathcal{F}(\mu, \kappa)$ is the summand of an arbitrary coefficient $c_{i, j}(\mu)$ from (4).

\section{Proof of the First Theorem}

First, we observe that a change of variable $y:=1-x$ is useful when expanding the denominator of the right hand side of the identity (1),

$$
\left(1-2 t x+t^{2}\right)^{n-1}=\sum_{l=0}^{n-1}\left(\begin{array}{c}
n-1 \\
l
\end{array}\right)(2 t y)^{n-1-l}(1-t)^{2 l} .
$$

In view of this substitution, it is convenient to use the following representation for the Gegenbauer polynomials ([1], 6.4.9 and 6.3.5),

$$
C_{k}^{\frac{n-2}{2}}(x)=\left(\begin{array}{c}
k+n-3 \\
k
\end{array}\right){ }_{2} F_{1}\left(\begin{array}{c}
-k, k+n-2 \\
\frac{n-1}{2}
\end{array} ; \frac{1-x}{2}\right) .
$$

Using (8) and multiplying both sides of the identity (1) with the expression (7), it remains to prove that

$$
\begin{gathered}
\sum_{k \geq 0} \frac{\left(\begin{array}{c}
k+n-2 \\
k
\end{array}\right)\left(\begin{array}{c}
k+n-3 \\
k
\end{array}\right)}{\left(\begin{array}{c}
k+\frac{n}{2}-2 \\
k
\end{array}\right)} t^{k} \sum_{j \geq 0} \frac{(k)_{j}\left(1-\frac{n}{2}\right)_{j}}{\left(k+\frac{n}{2}\right)_{j} \Gamma(j+1)} t^{2 j} \sum_{i \geq 0} \frac{(-k)_{i}(k+n-2)_{i}}{\left(\frac{n-1}{2}\right)_{i} \Gamma(i+1) 2^{i}} y^{i} \\
\times \sum_{l=0}^{n-1}\left(\begin{array}{c}
n-1 \\
l
\end{array}\right)(2 t y)^{n-1-l}(1-t)^{2 l}=\left(1-t^{2}\right)^{n-1}
\end{gathered}
$$


holds for all $n \in \mathbb{N}$ with $n \geq 3$ and for all real variables $t, y$ with $|t|<1$ and $0<y<2$.

Remark: In [5] it was proven that for every fixed $t$ with $|t|<1$ the left hand side of (1) is a uniformly convergent series in the interval $-1 \leq x \leq 1$. Hence, we can proceed with the coefficient comparison with respect to the new variable $y$; see also ([9], 3.32).

One needs to deal with the constant coefficient with respect to $y$ separately, so we continue with the following case distinction, cases (a) and (b).

(a) In the multisum expression on the left hand side of (9), the constant coefficient with respect to $y$ is obtained when $l=n-1$ and $i=0$. Consequently, this case reduces to proving that

$$
\sum_{k \geq 0} \frac{\left(\begin{array}{c}
k+n-2 \\
k
\end{array}\right)\left(\begin{array}{c}
k+n-3 \\
k
\end{array}\right)}{\left(\begin{array}{c}
k+\frac{n}{2}-2 \\
k
\end{array}\right)} t^{k} \sum_{j \geq 0} \frac{(k)_{j}\left(1-\frac{n}{2}\right)_{j}}{\left(k+\frac{n}{2}\right)_{j} \Gamma(j+1)} t^{2 j}=\left(\frac{1+t}{1-t}\right)^{n-1}
$$

holds for all $|t|<1$ and $n \geq 3$.

Furthermore, using the binomial theorem, the right hand side of (10) can be written as

$$
\left(\frac{1+t}{1-t}\right)^{n-1}=\sum_{m \geq 0}(-t)^{m} \sum_{s=0}^{m}\left(\begin{array}{c}
n-1 \\
s
\end{array}\right)\left(\begin{array}{c}
-n+1 \\
m-s
\end{array}\right)(-1)^{s} .
$$

Via coefficient comparison with respect to $t^{m}$ for an arbitrary $m \geq 0$, we obtain the equality of two single sum expressions

$$
\left.\sum_{j \geq 0} \frac{\left(\begin{array}{c}
k+n-2 \\
k
\end{array}\right)\left(\begin{array}{c}
k+n-3 \\
k
\end{array}\right)}{\left(\begin{array}{c}
k+\frac{n}{2}-2 \\
k
\end{array}\right)} \frac{(k)_{j}\left(1-\frac{n}{2}\right)_{j}}{\left(k+\frac{n}{2}\right)_{j} \Gamma(j+1)}\right|_{k=m-2 j}=(-1)^{m} \sum_{s=0}^{m}\left(\begin{array}{c}
n-1 \\
s
\end{array}\right)\left(\begin{array}{c}
-n+1 \\
m-s
\end{array}\right)(-1)^{s} .
$$

Note here that both sides of this last identity are terminating sums and that in classical hypergeometric notation, we relate a ${ }_{7} F_{6}$ to a ${ }_{2} F_{1}$ series. However, proceeding algorithmically we prefer to use Zeilberger's algorithm to prove this identity. The Mathematica implementation [4] delivers the same recurrence,

$$
m \mathrm{SUM}[m]+2(n-1) \operatorname{SUM}[1+m]-(2+m) \operatorname{SUM}[2+m]=0
$$

for both sides of (11). At last, it is trivial to check that the identity (11) holds for $m=0$ and $m=1$.

(b) We also need to show that the coefficients of all the powers $r \geq 1$ of the real variable $y$ are zero. To determine the coefficient of $y^{r}$ with $r \geq 1$, we choose in the multisum on the left hand side of (9) the term where $l=n-1+i-r$. Consequently, the following identity must hold

$$
\begin{aligned}
& \sum_{k \geq 0} \frac{\left(\begin{array}{c}
k+n-2 \\
k
\end{array}\right)\left(\begin{array}{c}
k+n-3 \\
k
\end{array}\right)}{\left(\begin{array}{c}
k+\frac{n}{2}-2 \\
k
\end{array}\right)} t^{k} \sum_{j \geq 0} \frac{(k)_{j}\left(1-\frac{n}{2}\right)_{j}}{\left(k+\frac{n}{2}\right)_{j} \Gamma(j+1)} t^{2 j} \\
& \quad \times \sum_{i=0}^{r} \frac{(-k)_{i}(k+n-2)_{i}}{\left(\frac{n-1}{2}\right)_{i} \Gamma(i+1) 4^{i}}\left(\begin{array}{c}
n-1 \\
r-i
\end{array}\right) t^{r-i}(1-t)^{2 i}=0
\end{aligned}
$$

for $|t|<1$ and integers $r \geq 1, n \geq 3$. 
Moreover, in (12) the coefficient of an arbitrary power $p \geq 0$ of the variable $t$ must be zero. Therefore, we will prove that

$$
\begin{aligned}
& \sum_{k \geq 0} \frac{\left(\begin{array}{c}
k+n-2 \\
k
\end{array}\right)\left(\begin{array}{c}
k+n-3 \\
k
\end{array}\right)}{\left(\begin{array}{c}
k+\frac{n}{2}-2 \\
k
\end{array}\right)} \sum_{j \geq 0} \frac{(k)_{j}\left(1-\frac{n}{2}\right)_{j}}{\left(k+\frac{n}{2}\right)_{j} \Gamma(j+1)} \\
& \quad \times \sum_{i=0}^{r} \frac{(-k)_{i}(k+n-2)_{i}}{\left(\frac{n-1}{2}\right)_{i} \Gamma(i+1) 4^{i}}\left(\begin{array}{c}
n-1 \\
r-i
\end{array}\right)\left(\begin{array}{c}
2 i \\
p-r+i-k-2 j
\end{array}\right)(-1)^{i-k}=0
\end{aligned}
$$

holds for all integer variables $r \geq 1, p \geq 0$ and $n \geq 3$.

Because there is no obvious further simplification of this expression, we now algorithmically compute a recurrence satisfied by the left hand side of (13). The triple sum in (13) comes in three parameters; to indicate this explicitly we denote it as a function of $r, p$ and $n$,

$$
S[r, p, n]:=\sum_{k \geq 0} \sum_{j \geq 0} \sum_{i \geq 0} F[r, p, n, k, j, i] .
$$

Since we will only search for a recurrence in the parameters $r$ and $p$, we view the triple sum (14) as a function $\operatorname{SUM}[\cdot, \cdot]$ defined on lattice points from $\{r \in \mathbb{Z}: r \geq 1\} \times\{p \in \mathbb{Z}: p \geq 0\}$. Note that, the value of $\operatorname{SUM}[f, p]$ at an arbitrary integer lattice point is a finite sum and is dependent of the integer variable $n \geq 3$.

Remark: Because the new integer variables, $r$ and $p$, have been introduced when comparing coefficients, finding a recurrence and showing that sufficiently many initial values are zero, corresponds to proving that in (9) the coefficients of $y^{r} t^{p}$ for all $r \geq 1$ and $p \geq 0$ are zero. This proof strategy, motivated by the induction principle, has also a significant computational advantage since the sums arising from the coefficient comparison are finite.

As it was mentioned in the introduction, before applying Wegschaider's algorithm [6] we determine a suitable set of shifts, called structure set, for the desired recurrence. An algorithm for computing small structure sets is implemented in the Mathematica package MultiSum ${ }^{1}$; see also [2]. The following command determines 8 candidate structure sets for a recurrence in $r$ and $p$ satisfied by the summand $F[r, p, n, k, j, i]$ of the triple sum from (13):

$\ln [2]:=$ FindStructureSet $[F[r, p, n, k, j, i],\{r, p\},\{0,0\},\{k, j, i\},\{0,1,1\}, 1]$

Settling, for instance, on the first candidate structure set, Wegschaider's algorithm [6] computes a recurrence for the summand $F[r, p, n, k, j, i]$, called certificate recurrence. In less then 300 seconds on an average personal computer one obtains a recurrence for the summand as output of the command

$\ln [3]:=$ FindRecurrence $[F[r, p, n, k, j, i],\{r, p\},\{k, j, i\}, \%[[1]], 1, \mathrm{WZ} \rightarrow$ True $]$.

The certificate recurrence has as coefficients polynomials free of the summation variables $k$, $j$ and $i$. Therefore, by summing over the certificate recurrence we obtain the desired recurrence for the triple sum $\operatorname{SUM}[r, p]$ from (13). This is done automatically by the command

$$
\begin{aligned}
\operatorname{In}[4]:= & \text { SumCertificate }[\%] \\
\text { Out }[4]= & \left\{-(n+p-3 r)(p-r) \operatorname{SUM}[r, p]+\left(2 n^{2}+3 p n-9 r n-n-p^{2}+5 r^{2}-6 p+10 r-3\right)\right. \\
& \operatorname{SUM}[r, p+1]+\left(-2 n^{2}+3 p n+3 r n+10 n+p^{2}-r^{2}-4 p r-10 r-6\right) \operatorname{SUM}[r, p+2] \\
& -(n-p-r-3)(p-r+3) \operatorname{SUM}[r, p+3]+(p-r)(n-p+3 r) \operatorname{SUM}[r+1, p+1] \\
& +\left(-2 n^{2}+p n-5 r n+n-p^{2}+9 r^{2}-6 p+20 r+1\right) \operatorname{SUM}[r+1, p+2]+ \\
& \left(2 n^{2}+p n+3 r n+2 n+p^{2}-5 r^{2}-4 p r-20 r-10\right) \operatorname{SUM}[r+1, p+3]+
\end{aligned}
$$

\footnotetext{
$1_{\text {available at http://www.risc.uni-linz.ac.at/research/combinat/software/ }}$
} 


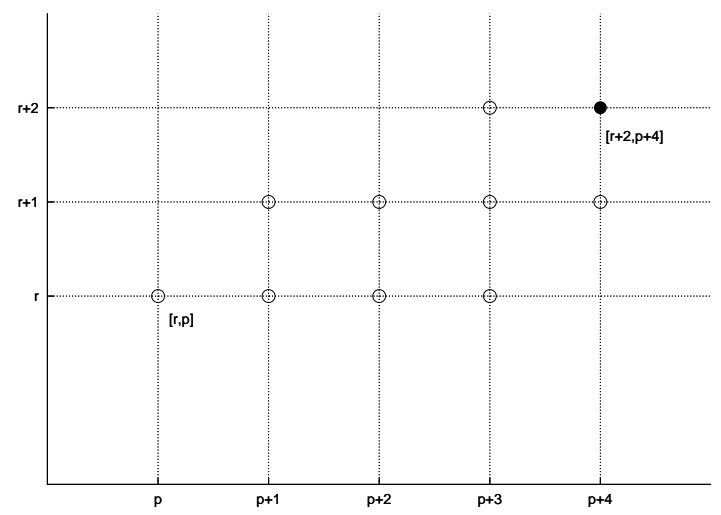

(a) The multivariate recurrence

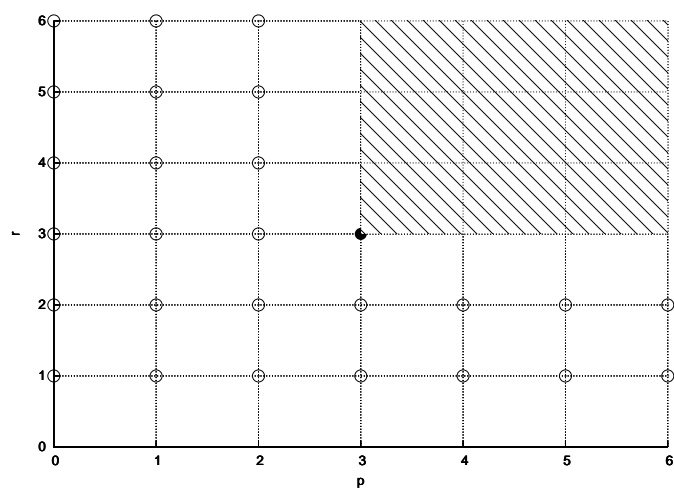

(b) The initial values

Figure 1

$$
\begin{aligned}
& (p-r+3)(n+p+r+3) \operatorname{SUM}[r+1, p+4]+2(r+2)(n+2 r+1) \\
& \operatorname{SUM}[r+2, p+3]-2(r+2)(n+2 r+1) \operatorname{SUM}[r+2, p+4]=0\} .
\end{aligned}
$$

One can see that the value of $\operatorname{SUM}[r+2, p+4]$ can be computed from the recurrence if the values of the function $\operatorname{SUM}[\cdot, \cdot]$ in all the integer lattice points marked with $\circ$ in Figure 1 (a) are known.

After choosing the leading term, we observe that its coefficient in the recurrence is non-zero for all positive values of $r$ and $n$. Having a visualization of the recurrence at hand, it is also clear which initial values need to be checked; see Figure 1(b). For an arbitrary $p \geq 0$ we need to show that the triple sum from (13) is zero in case $r=1$ and $r=2$; while for a fixed $r \geq 1$ we need to check the cases when $p \in\{0,1,2\}$. It is trivial to see that all the initial values are zero, since in all five cases we only need to compute finite sums with summation bounds being fixed integers.

\section{Proof of the Second Theorem}

Since $|t|<1$, the following quadratic transformation that goes back to Gauss ([1], 3.1.3)

$$
{ }_{2} F_{1}\left(\begin{array}{c}
k, k+n-1 \\
k+\frac{n}{2}
\end{array} ; \frac{1-\sqrt{1-t^{2}}}{2}\right)={ }_{2} F_{1}\left(\begin{array}{c}
\frac{k}{2}, \frac{k+n-1}{2} ; \\
k+\frac{n}{2}
\end{array} ; t^{2}\right)
$$

can be applied to the identity (2).

Furthermore, we use the representation (3) for the Gegenbauer polynomials and the identity (2) is brought to a more convenient form for the purpose of coefficient comparison:

$$
\begin{gathered}
\sum_{k \geq 0} \frac{\Gamma\left(\frac{k+n}{2}\right) \Gamma\left(\frac{k+1}{2}\right)}{\Gamma(k+1)}(2 x t)^{k}{ }_{2} F_{1}\left(\begin{array}{c}
\frac{k}{2}, \frac{k+n-1}{2}{ }_{k+\frac{n}{2}}^{2} ; t^{2}
\end{array}\right){ }_{2} F_{1}\left(\begin{array}{c}
-\frac{k}{2}, \frac{1-k}{2} ; \\
2-k-\frac{n}{2}
\end{array} ; \frac{1}{x^{2}}\right) \\
=\frac{2}{n} \Gamma\left(\frac{n+1}{2}\right) \sqrt{1-t^{2}}{ }_{2} F_{1}\left(\begin{array}{c}
n, 1 ; \\
\frac{n}{2}+1
\end{array} ; \frac{x t+1}{2}\right) .
\end{gathered}
$$


Thus, we will prove that

$$
\begin{aligned}
\sum_{k \geq 0} \frac{\Gamma\left(\frac{k+n}{2}\right) \Gamma\left(\frac{k+1}{2}\right)}{\Gamma(k+1)} & (2 x t)^{k} \sum_{j \geq 0} \frac{\left(\frac{k}{2}\right)_{j}\left(\frac{k+n-1}{2}\right)_{j}}{\left(\frac{n}{2}+k\right)_{j} \Gamma(j+1)} t^{2 j} \sum_{i \geq 0} \frac{\left(-\frac{k}{2}\right)_{i}\left(\frac{1-k}{2}\right)_{i}}{\left(2-\frac{n}{2}-k\right)_{i} \Gamma(i+1)} x^{-2 i} \\
= & \frac{2}{n} \Gamma\left(\frac{n+1}{2}\right) \sqrt{1-t^{2}} \sum_{s \geq 0} \frac{(n)_{s}}{\left(\frac{n}{2}+1\right)_{s} 2^{s}}(1+x t)^{s}
\end{aligned}
$$

holds for all $|x|<1,|t|<1$ and $n \in \mathbb{N}$ with $n \geq 3$.

After exchanging the order of summation, on the left hand side of (15) the coefficient of $x^{m}$ for any $m \geq 0$, can be determined by setting $k=m+2 i$. Comparing coefficients with respect to $x^{m}$ on both sides of (15), reduces the problem to showing that

$$
\begin{aligned}
& \sum_{i \geq 0} \frac{\Gamma\left(\frac{k+n}{2}\right) \Gamma\left(\frac{k+1}{2}\right)}{\Gamma(k+1)}\left.(2 t)^{k} \frac{\left(-\frac{k}{2}\right)_{i}\left(\frac{1-k}{2}\right)_{i}}{\left(2-\frac{n}{2}-k\right)_{i} \Gamma(i+1)} \sum_{j \geq 0} \frac{\left(\frac{k}{2}\right)_{j}\left(\frac{k+n-1}{2}\right)_{j}}{\left(\frac{n}{2}+k\right)_{j} \Gamma(j+1)} t^{2 j}\right|_{k=m+2 i} \\
&=\frac{2}{n} \Gamma\left(\frac{n+1}{2}\right) t^{m} \sqrt{1-t^{2}} \sum_{s \geq m}\left(\begin{array}{c}
s \\
m
\end{array}\right) \frac{(n)_{s}}{\left(\frac{n}{2}+1\right)_{s} 2^{s}}
\end{aligned}
$$

holds for all integers $m \geq 0, n \geq 3$ and real $|t|<1$.

Using the second order recurrence in the parameter $m \geq 0$ delivered by Zeilberger's algorithm [8], we can easily compute a closed form for the non-terminating sum on the right hand side of (16). Plugging this in, (16) is equivalent to

$$
\begin{gathered}
\left.\sum_{i \geq 0} \frac{\Gamma\left(\frac{k+n}{2}\right) \Gamma\left(\frac{k+1}{2}\right)}{\Gamma(k+1)} 2^{k} t^{2 i} \frac{\left(-\frac{k}{2}\right)_{i}\left(\frac{1-k}{2}\right)_{i}}{\left(2-\frac{n}{2}-k\right)_{i} \Gamma(i+1)} \sum_{j \geq 0} \frac{\left(\frac{k}{2}\right)_{j}\left(\frac{k+n-1}{2}\right)_{j}}{\left(\frac{n}{2}+k\right)_{j} \Gamma(j+1)} t^{2 j}\right|_{k=m+2 i} \\
=\sqrt{\pi} \frac{\Gamma\left(\frac{m+n}{2}\right)}{\Gamma\left(1+\frac{m}{2}\right)} \sum_{q \geq 0}\left(\begin{array}{c}
1 / 2 \\
q
\end{array}\right)\left(-t^{2}\right)^{q} .
\end{gathered}
$$

On the left hand side of (17) the coefficient of an arbitrary power $q \geq 0$ of $t^{2}$ is obtained by setting $j=q-i$. Comparing the coefficients of $t^{2 q}$ in (17) leads to the following identity

$$
\begin{aligned}
\sum_{i \geq 0} \frac{\Gamma\left(\frac{k+n}{2}\right) \Gamma\left(\frac{k+1}{2}\right)}{\Gamma(k+1)} 2^{k} & \left.\frac{\left(-\frac{k}{2}\right)_{i}\left(\frac{1-k}{2}\right)_{i}}{\left(2-\frac{n}{2}-k\right)_{i} \Gamma(i+1)} \frac{\left(\frac{k}{2}\right)_{j}\left(\frac{k+n-1}{2}\right)_{j}}{\left(\frac{n}{2}+k\right)_{j} \Gamma(j+1)}\right|_{\substack{k=m+2 i \\
j=q-i}} \\
& =\sqrt{\pi} \frac{\Gamma\left(\frac{m+n}{2}\right)}{\Gamma\left(1+\frac{m}{2}\right)}(-1)^{q}\left(\begin{array}{c}
1 / 2 \\
q
\end{array}\right) .
\end{aligned}
$$

To prove that (18) holds for all integers $q \geq 0$ and $n \geq 3$, we use again Zeilberger's algorithm [8]. The Mathematica implementation ${ }^{2}$ described in [4] delivers a first order recurrence for the single terminating sum on the left hand side of (18):

$$
(2 q-1) \operatorname{SUM}[q]-2(q+1) \operatorname{SUM}[q+1]=0 .
$$

We can easily check that the expression on the right hand side of (18) also satisfies this output recurrence. At last, we verify the identity (18) at the initial value $q=0$ and we obtain

$$
2^{m} \Gamma\left(\frac{m+1}{2}\right) \Gamma\left(\frac{m}{2}+1\right)=\sqrt{\pi} \Gamma(m+1) .
$$

\footnotetext{
2 available at http://www.risc.uni-linz.ac.at/research/combinat/software/
} 
The identity (19) is known as Legendre's duplication formula ([1], 1.5) and it holds for all $m \geq 0$. Herewith, the proof of the Theorem 2 is complete.

\section{Conclusion}

We use WZ-summation methods to prove two theorems that go back to the work of Symeonidis [5]. These are, to our knowledge, the first direct proofs of the identities (1) and (2).

Our method of proof uses coefficient comparisons with respect to certain parameters to reduce the complexity of the identities. Coefficient comparisons lead to case distinctions, but in most cases we are left with single sum expressions and because of the structure of the identities, Zeilberger's algorithm [8] can be applied. In the situations where nested multisums remain, we can use Wegschaider's algorithm [6] to compute recurrences for both sides of the reduced identity. To complete the proofs we only need to check finitely many initial values.

Note that both algorithms delivered recurrences for the summand of the given sums $\sum_{\kappa} \mathcal{F}(\mu, \kappa)$. Summing the certificate recurrence over all the variables from $\kappa$, we obtained the desired recurrences for the sum. In each case one can easily check that the algorithmically computed certificate recurrence holds, so the algorithms also deliver a proof for the recurrence satisfied by the sum.

On the other hand there are several non algorithmic aspects involved in the proofs. In the case of Theorem 1, a change of variables is necessary. The proof of Theorem 2 becomes straight-forward after applying a quadratic transformation to one of the hypergeometric terms involved. Also convergence issues, like absolute convergence for exchanging the order of summation, needed to be considered at various steps of the proofs. We have omitted these details that can be supplied by routine analysis.

Acknowledgement. We thank Prof. Peter Paule for helpful comments and suggestions. Special thanks go to our colleague Carsten Schneider who checked with his Sigma package some of our computations.

\section{REFERENCES}

[1] G.E. Andrews, R. Askey, R. Roy. Special Functions, volume 71 of Encyclopedia of Mathematics and its Applications. Cambridge University Press, 1999.

[2] R. Lyons, P. Paule and A. Riese. A computer proof of a series evaluation in terms of harmonic numbers, Appl. Algebra Engrg. Comm. Comput., 13: 327-333, 2002.

[3] M. Muldoon. The electronic news net of the SIAM activity group on orthogonal polynomials and special functions, 2004. http://staff.science.uva.nl/ thk/opsfnet/11.2.

[4] P. Paule and M. Schorn. A Mathematica version of Zeilberger's algorithm for proving binomial coefficient identities. Journal of Symbolic Computation, 20(5-6):673-698, 1995.

[5] E. Symeonidis. The Poisson integral for a ball in spaces of constant curvature. Comment. Math. Univ. Carolinae, 44(3):437-460, 2003.

[6] K. Wegschaider. Computer generated proofs of binomial multi-sum identities. Master's thesis, RISC-Linz, May 1997. http://www.risc.uni-linz.ac.at/research/combinat/publications

[7] H.S. Wilf and D. Zeilberger. An algorithmic proof theory for hypergeometric (ordinary and $q$ ) multisum/integral identities. Inventiones mathematicae, 108:575-633, 1992.

[8] D. Zeilberger. A fast algorithm for proving terminating hypergeometric identities. Discrete Mathematics, 80:207-211, 1990.

[9] E.T. Whittaker, G.N. Watson. A Course of modern analysis. Cambridge University Press, 1927 